\title{
Mouse and human mitochondrial nucleoid - detailed structure in relation to function
}

\author{
Jarmil Prachař \\ Laboratory of Electron Microscopy, Cancer Research Institute, Slovak Academy of Sciences, Vlárska 7, 83391 Bratislava, Slovakia
}

\begin{abstract}
The independent mitochondrial genetic information is organized in so-called mitochondrial nucleoids that, in vertebrates, typically contain 5-7 genetic units. The total number of nucleoids per cell is several hundred in cultured cells. Mitochondrial nucleoids, similarly to the whole mitochondrial network, have recently been successfully and extensively visualized using fluorescent and confocal microscopy. In the present work, we show high-resolution micrographs of mouse and human mitochondrial nucleoids obtained by transmission electron microscopy. Position in the mitochondria, size, general appearance and other properties of the human nucleoids appear the same as those of mouse nucleoids, and all observations are also in full agreement with the results obtained in different laboratories using different approaches. Most of nucleoids are located inside mitochondrial tubes. However, we show directly that certain part of the nucleoids close to inner membrane is bound to the complex of molecules that crosscut both, the inner and the outer mitochondrial membranes. Nucleoids in cells starving for serum are mostly more dense than those in dividing cells. We discuss the position, appearance and other properties of the nucleoids in relation to functional stage. Other electron-dense structures inside mitochondria that could be erroneously considered to be mitochondrial nucleoids are also described.
\end{abstract}

Key words: Mitochondrion — Mitochondrial nucleoid — Mouse and human cells — Ultrastructure - Transmission electron microscopy

\section{Introduction}

Most of the genetic information that codes for mitochondrial proteins is located in the nuclear chromosomes. Therefore, most of the proteins have origins in the mRNAs transcribed from nuclear genes and translated on cytoplasmic ribosomes. Proteins destined for mitochondria are transported by sophisticated means through one or two membranes inside these organelles. However, an important small fraction of genetic information is maintained independently of the nucleus in the mitochondrial matrix. This information is organized in about 450-800 mitochondrial nucleoids (in further text only nucleoids), in in vitro cultured cells (Iborra et al. 2004; Legros et al. 2004). Recent research on nucleoids has been directed especially

Correspondence to: Jarmil Prachař, Laboratory of Electron Microscopy, Cancer Research Institute, Slovak Academy of Sciences, Vlárska 7, 83391 Bratislava, Slovakia

E-mail: jarmil.prachar@savba.sk at determining their protein composition and, from the presence of these proteins, functional relationships are deduced (Nosek et al. 2006; Wang and Bogenhagen 2006; Chen et al. 2007; He et al. 2007; Holt et al. 2007; Kaufman et al. 2007; Bogenhagen et al. 2008; Macierzanka et al. 2008; Sumitani et al. 2009).

To this date, there exist only rare micrographs from early mitochondrial research showing nucleoids, acquired by means of transmission electron microscopy (Nass 1969), but those micrographs are of low resolution, corresponding to the capabilities of electron microscopes at that time and the different microscopy strategies. The nucleoid was documented by immune-electron microscopy in one study only (Iborra et al. 2004).

In the course of electron microscopy studies of mitochondrial contact with the nucleus (Prachar 2003), some sections of the mitochondria containing small electron-dense objects were observed and studied in more detail by using high-resolution transmission electron microscopy. Through comparison with the published data, we concluded that 
these objects are unambiguously mitochondrial nucleoids. The nucleoids are seen as approximately spherical particles composed of thin filaments. Certain filaments protrude from the bodies and serve to anchor in the membranes. The nucleoids behave similarly to any other nucleoprotein; they are observed as electron-dense ball-shaped bodies of apparently the same diameter, as was calculated from the confocal microscopy results.

At present, there exists only one organism whose mitochondrial nucleoids have been seriously morphologically characterized using transmission electron microscopy - the lower eukaryotic species, Physarum polycephalum (Suzuki et al. 1982; Kuroiwa et al. 2006). The mitochondrial nucleoids of the true slime mold (myxomycete), Physarum, and of a species of Myogastria were characterized as large electron-dense structures situated in a central region of the inner matrix of spherical mitochondria (Kuroiwa 1973). The nucleoid of Physarum is different from the nucleoids of higher eukaryotes in terms of size and shape (Sasaki et al. 2003).

Present data, obtained in the Laboratory of Electron Microscopy, show high-resolution micrographs of mouse and human mitochondrial nucleoids in several positions in the mitochondria of cells growing, in the presence of serum and in cells starving for serum. We discuss why it is more difficult to find nucleoids in the mitochondria of the human cell lines used than in the mitochondria of mouse cells. The fine structure of the nucleoids is also shown.

This work is especially devoted to showing strategies and clues for researchers on how transmission electron microscopy can be used to look for and detect mitochondrial nucleoids in cell sections.

\section{Materials and Methods}

\section{Cell cultures}

Mouse leukemia cell line L1210 (purchased from the American Type Culture Collection, Rockville, MD, USA) was maintained in RPMI 1640 medium supplemented with $10 \%$ fetal bovine serum. The cells were fixed at a cell density of $10^{6} / \mathrm{ml}$ while still dividing but after the exponential growth phase.

One part of the L1210 cells was taken at a density of $5 \times$ $105 / \mathrm{ml}$ (exponential phase), washed one time by centrifugation in RPMI 1640 without serum, seeded into RPMI 1640 without serum and cultured for the next $24 \mathrm{~h}$. These cells are referred to as cells starving for serum.

The MT1 cell line (mutated hMSH6) is mismatch repair deficient, derived from the parental human lymphoblastoid TK6 cell line, and was kindly provided by J. Jiricny (Institute of Molecular Cancer Research, Zürich, Switzerland).
Cells were routinely cultured in RPMI 1640 medium supplemented with $10 \%$ fetal bovine serum. The cells were fixed at a cell density of $10^{6} / \mathrm{ml}$ just after the exponential growth phase.

The human colon adenocarcinoma cell line Caco-2 was obtained from the European Collection of Cell Culture (Wiltshire, UK). The cells were cultured in RPMI 1640 medium supplemented with $10 \%$ fetal bovine serum and collected for electron microscopy study in the early exponential growth phase. The cells adhere very strongly to plastic and therefore glass dishes were used for cultivation to prevent loss of the bottom cellular membrane. The cells were scraped following osmium tetroxide postfixing.

\section{Preparation of samples for transmission electron microscopy}

The L1210 cells $(5 \mathrm{ml})$, and MT1 cells $(10 \mathrm{ml})$, were quickly pelletted $(2 \mathrm{~min})$ and immediately fixed without any washing. The Caco- 2 cells were treated the same way as the leukemia cells, but after the postfixing step (see above). Fixation, contrasting, embedding and staining of sections were done by conventional procedures. Briefly, the procedure was as follows: fixation with $3 \%$ glutaraldehyde in PBS for $1 \mathrm{~h}$, postfixation in $1 \% \mathrm{OsO}_{4}$ for $1 \mathrm{~h}$, washing 3 times with cold deionized water, and contrasting with a saturated solution of uranyl acetate in deionized water overnight. For washing, cells were resuspended in water and shortly centrifuged 3 times between all steps. Subsequently, the cells were resuspended in low melting point agarose $\left(1 \%\right.$ in water) at $37^{\circ} \mathrm{C}$, pelletted and kept in an ice bath for a short time; this is the embedment in agarose. The following step was dehydration in $70 \%$ and $100 \%$ ethyl alcohol and equilibration in propylene oxide. Embedding was done in the Poly/Bed 812 epoxy resin (Polysciences). For preparation of the epoxy resin, a combination of components was chosen to achieve maximal hardness, according to the manufacturer's instructions. The sample in epoxy resin was kept at $37^{\circ} \mathrm{C}$ for $48 \mathrm{~h}$ to harden, and at $50^{\circ} \mathrm{C}$ for the next $24 \mathrm{~h}$. Ultrathin sections were picked up directly (without any supporting membrane) on 700 or 1000 mesh copper grids (Agar Scientific). Section thicknesses were about $50 \mathrm{~nm}$ or less. Contrasting of the ultrathin sections (one side only) was done on a drop of a saturated solution of uranyl acetate in water at $37^{\circ} \mathrm{C}(2 \mathrm{~h})$ and, after intensive washing in deionized water, the last step was contrasting on a drop of Reynolds solution at laboratory temperature (4 min or less). Unless otherwise stated, all procedures were carried out at room temperature. Uranyl acetate and Reynolds solution were prepared the same day that contrasting was done.

Images were collected using the JEOL JEM-1200 EX transmission electron microscope at $80 \mathrm{kV}$ with Kodak electron microscope films 4489 . 


\section{Results}

Searching for nucleoids in the mouse leukemia cell line - the probability calculation of finding a nucleoid in the section

As mentioned in the Introduction, we first noticed mitochondrial nucleoids serendipitously.

The nucleoids are present as 450-800 distinct foci in cultured cells (Nass 1969; Iborra et al. 2004; Legros et al. 2004). The cell diameter of L1210 mouse leukemia cells is about $12 \mu \mathrm{m}$. The thickness of our sections is usually about $50 \mathrm{~nm}$. Therefore, one cell is cut into 240 sections. For calculation, we took the lower estimate of the nucleoid number per cell, which is about 480 (Iborra et al. 2004). Therefore, in each average cell section we should find two nucleoids. This is a very rough estimate, because there is a higher probability of finding nucleoids in the sections of larger area. On the other hand, in the section through the middle of the cell, the nucleus occupies a high proportion of the area. In addition, only part of the nucleoids can be seen, for methodological reasons (Fig. 1). In leukemia cells, the mitochondrial network is much less rich than that in epithelium-like cell lines. Despite these limitations, we supposed that at least one nucleoid could be found in at least one of the several cell sections. Because mitochondria are not evenly dispersed through the volume of the cell, we also supposed that, in some sections more rich in mitochondria, it should be possible to find more than one nucleoid. These estimates fit well for the L1210 mouse leukemia cells.

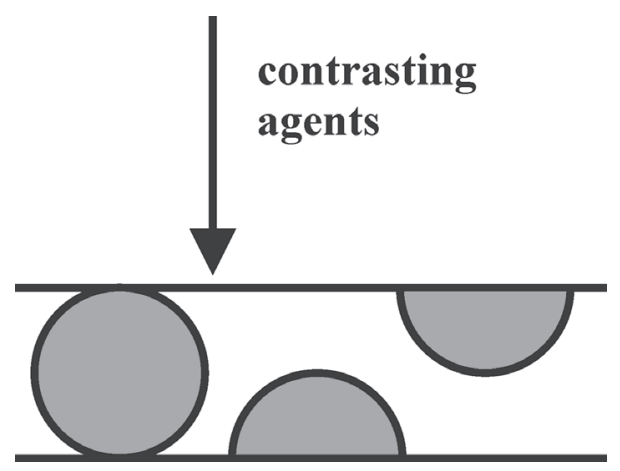

Figure 1. Outline of a random section through the cell. On average, one section will have two nucleoids randomly embedded (taken as a sum; one intact and two halves are in the present outline). To achieve higher resolution, the section is contrasted from one side only. It is likely that only the section through the nucleoid on the right hand side will show appropriate contrast and be registered by the observer because the negative charges on the surface are accessible to react with metal cations.
Nucleoids in the mitochondria of the L1210 mouse leukemia cells

We have found that more than $80 \%$ of the nucleoids are far from the inner surface of the mitochondria (300 nucleoids evaluated). As an example, we refer to one of the nucleoids in Fig. 2A. However, in this work, we documented micrographs especially of those nucleoids close to the inner surfaces of mitochondrial membranes. The reason for this is the presumption that nucleoids bound to the inner and outer membrane can have an important role in maintenance and replication (Shadel and Clayton 1997). Nucleoids were mostly seen in singles. Here, we often selected situations where two or more nucleoids were present to show more possibilities on one photograph.

In the field showing the cell in anaphase, we can see two nucleoids close to the inner surface of the mitochondrion and one in the middle of the mitochondrial tube (Fig. 2B).

A whole cell whose condensation of chromatin has begun is shown in Fig. 2C, where this cell is preparing for mitosis. The mitochondrial network is sectioned in three places with nucleoids. All three nucleoids are close to the inner mitochondrial surface.

A longitudinal section through a mitochondrion in Fig. 3A shows four nucleoids. The anchoring of the smallest nucleoid to the surface is documented in an enlargement of part of this figure (Fig. 3B). The electron-dense mass of the nucleoid does not touch the membranes. There is a zone of lower electron density; filaments anchoring the nucleoids in the membranes do not bind heavy metals as intensively as the nucleoid ball (due to the phosphate groups of DNA). Therefore, this anchoring is realized by protein filaments, and if DNA is present, it is present at a smaller proportion to the protein - less packed than in the nucleoid ball (see also Kondo-Okamoto et al. 2003). This nucleoid might represent one in the replication stage and the region of lower electron density might represent the replisome (Meeusen and Nunnari 2003). Anchorage, as such, might serve as a gateway for the transport of material from the cytoplasm to the newly forming nucleoid.

The shape of some nucleoids and the presence of stalks suggest that the nucleoid can be anchored in the membranes in more than one place (Fig. 4).

\section{Nucleoids in the mitochondria of L1210 mouse leukemia cells starving for serum}

The nucleoids in the cells starving for serum are observable even at a lower original magnification. They show strong contrast, because the matrix is much lighter than that of cells cultured in the presence of serum. The lighter appearance of the mitochondrial matrix is the result of low mitochondrial activity. Fig. 5 represents an example of a nucleoid that can be most easily noted by the observer. The very highly condensed nucleoid ball appears black on a very light background of the halo. 

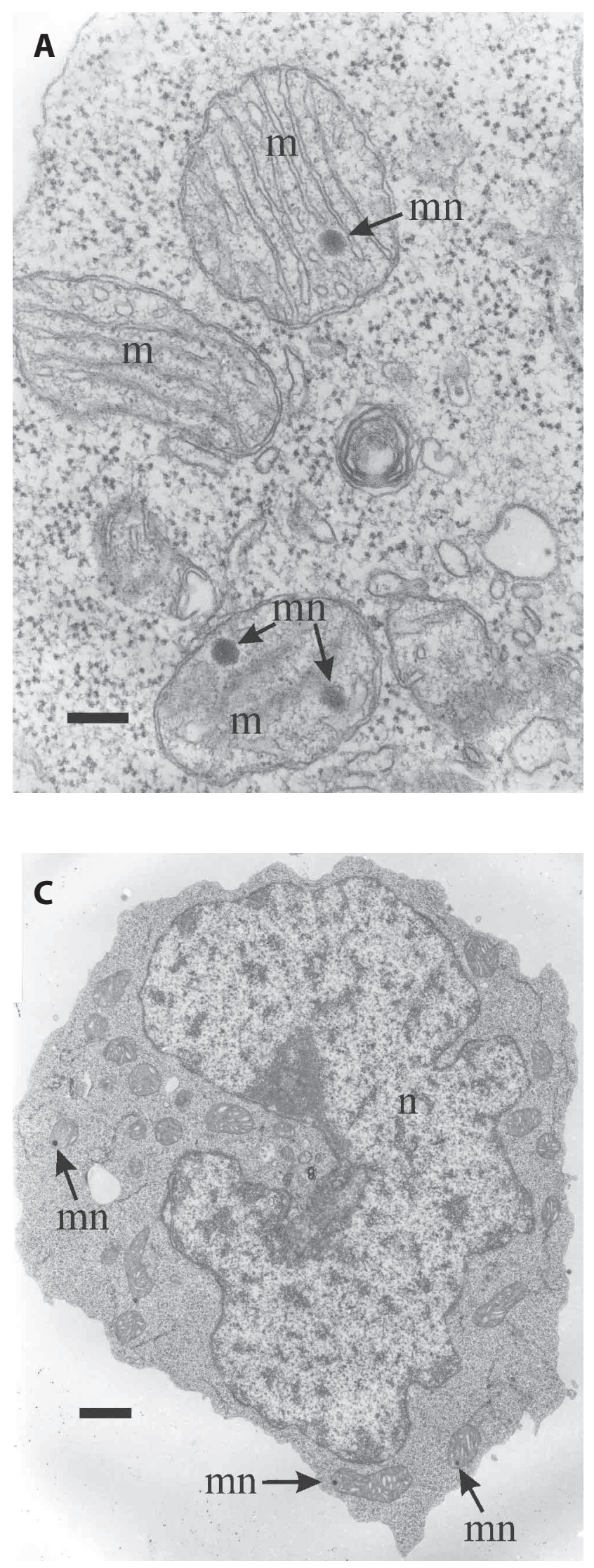

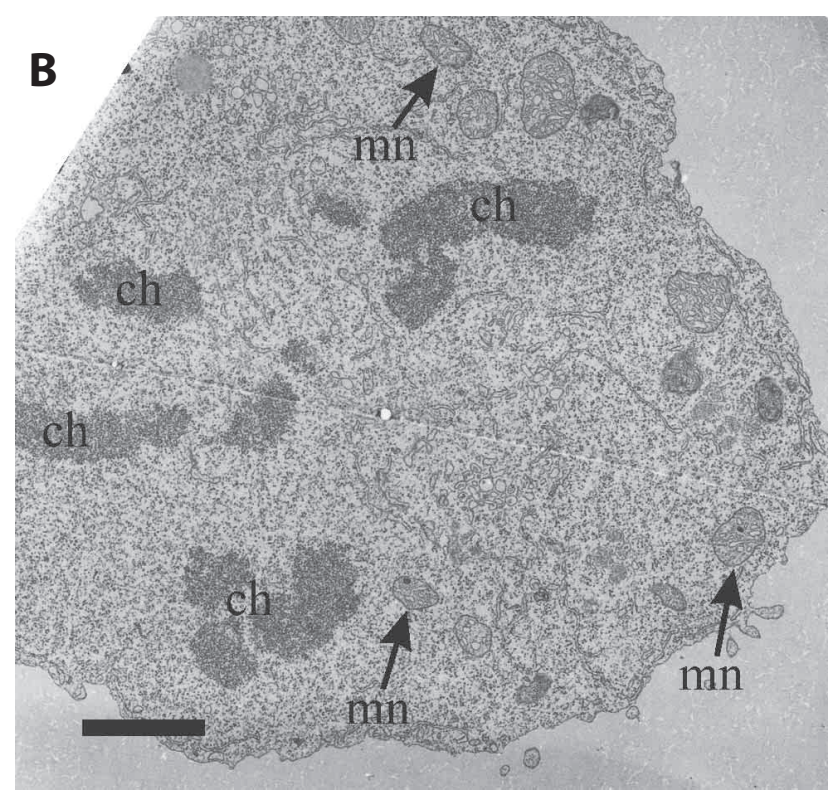

Frequency of nucleoid occurrence in human cells

It was more difficult to find nucleoids in the mitochondria of human cells than in mouse cells. This could be caused by an actual lower number of nucleoids, in the case of the human MT1 leukemia cells. In the case of the human colon carcinoma cell line, it could be caused by the presence of a more electron-dense mitochondrial matrix and the resultant inability to discern nucleoids blended with the surroundings. Eventually, however, after training and considerable patience, we found many nucleoids being in the same positions as in the mitochondria of mouse cells.

\section{Nucleoids in the mitochondria of Caco-2 human colon} carcinoma cells

Caco-2 cells are flat epithelial-like cells derived originally from a human colonic adenocarcinoma. These cells are

Figure 2. Localization of the nucleoids in different positions inside mitochondria in L1210 cells in various stages of cell cycle. A. The nucleoid in the upper part of the micrograph is apparently located inside the mitochondrial tube of the cell in interphase without contact with its surface. Magnification: $\times 30$ 000; scale bar: $200 \mathrm{~nm}$. B. Three sections of mitochondria of the cell in anaphase through nucleoid. Magnification: $\times 4000$; scale bar: $2 \mu \mathrm{m}$. C. Condensation of the chromatin before mitosis. Nucleoids are seen on section close to inner mitochondrial surface. Magnification: $\times 5000$; scale bar: $1 \mu \mathrm{m}$. $\mathrm{n}$, nucleus with dark patches of condensed chromatin around the inner membrane and also inside the nucleus; ch, sections through chromosomes; mn, nucleoids; arrows direct to the nucleoids or to the mitochondria containing nucleoids as a small dark dots in case of low original magnification. 


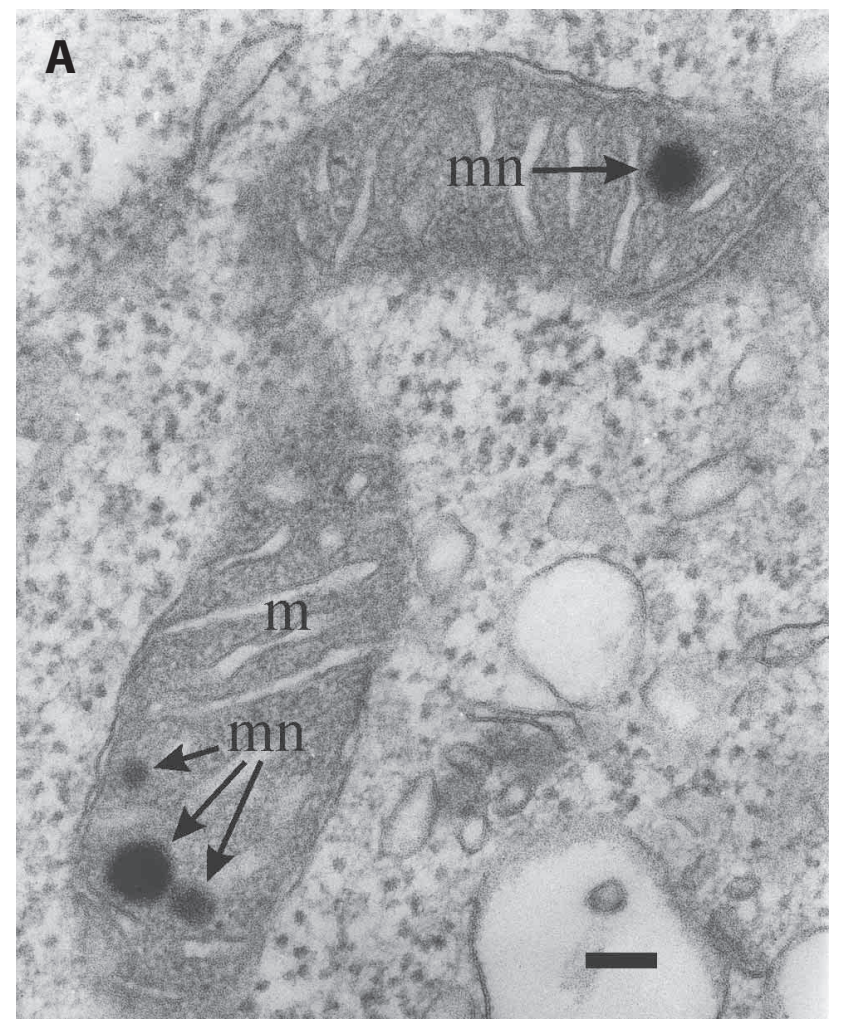

valuable in vitro tools for studies related to intestinal cell function and differentiation. The cells proliferate slowly in cell culture but manifest a high proteosynthetic activ-

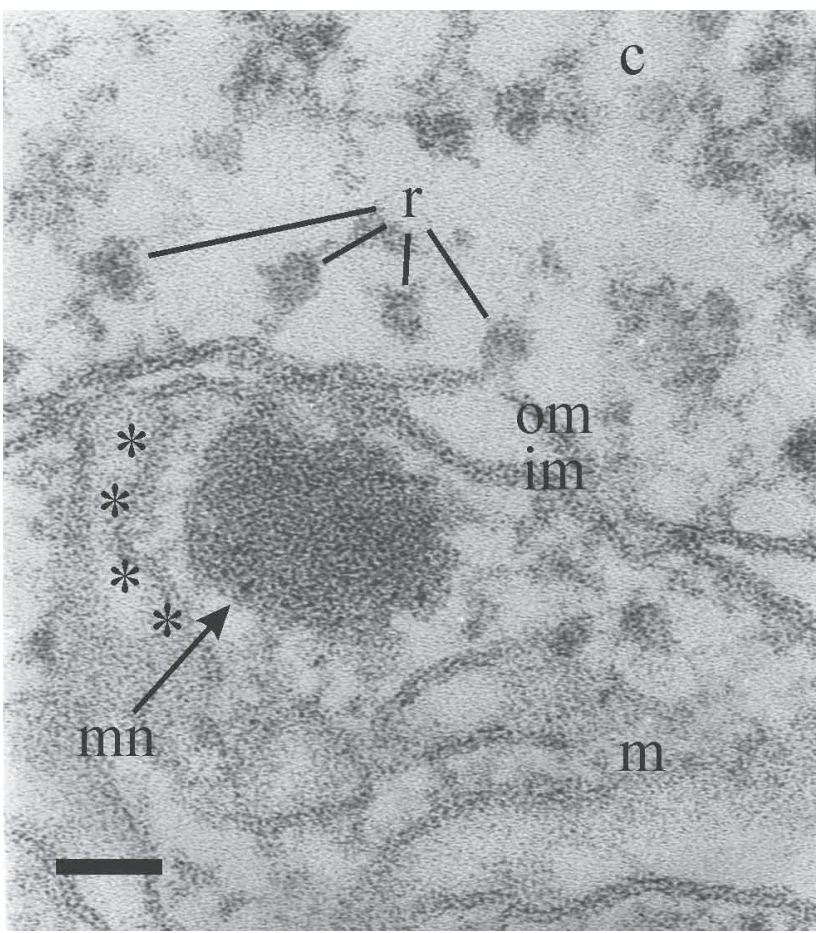

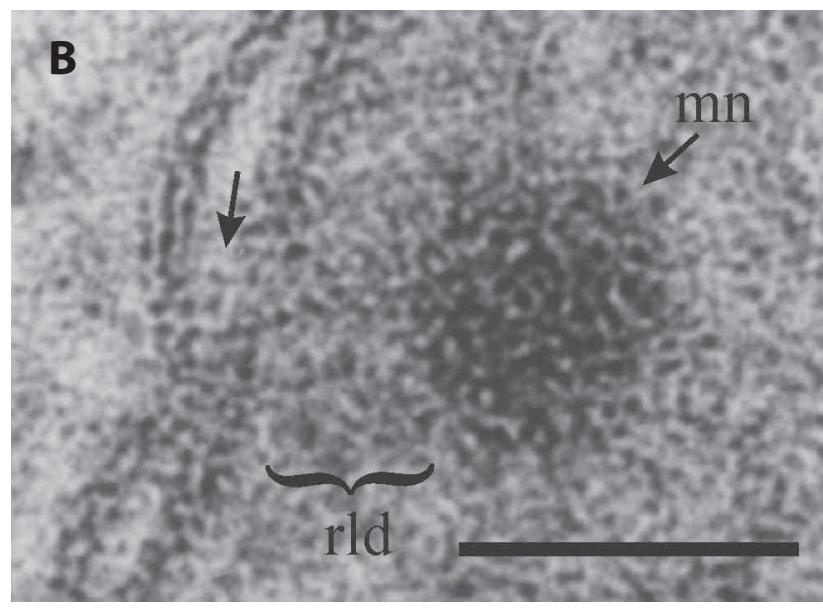

Figure 3. A. Mitochondrion in a L1210 cell with four nucleoids inside (extremely rare finding). Magnification: $\times 50$ 000; scale bar: $100 \mathrm{~nm}$. B. Enlargement of the same micrograph showing the region with the smallest nucleoid. Magnification: $\times 50000$; scale bar: $50 \mathrm{~nm}$; width of this bar: $2 \mathrm{~nm}$. $\mathrm{m}$, mitochondrion; $\mathrm{mn}$, nucleoids as dark balls; rld, region of lower electron density; arrow without letters is directed to the tapered region where filaments directing from the nucleoid are crossing both the inner and outer mitochondrial membranes. Strands of the biological molecules are light and dark heavy metals are around.

ity especially directed to extracellular production. They have a rich Golgi system, and a rich rough endoplasmic reticulum very often enveloping the mitochondria, and there are many different vesicles and vacuole-like spaces in the cytoplasm. The surface of the cell monolayer is slimy, likely because they produce mucous proteinous molecules as they originally did in the intestinal mucous membrane. Well-discernible subregions of nucleoli are one of the consequences of a strong proteosynthetic activity. Another consequence is a high mitochondrial activity manifested as an electron-dense matrix. This electron-dense background often precludes characterizing the surroundings of the nucleoids, for example the connection to the membranes. The general shape and basic components of the Caco-2 cell are shown in Fig. 6.

A nucleoid connected to the inner surface of the mitochondrion is presented in Fig. 7A.

4 Figure 4. Micrograph showing anchorage of the nucleoid to the inner and outer membranes of the L1210 cell mitochondrion (in two places in the upper part) and to cristae (in several places $-{ }^{*}$ ). Magnification: $\times 150$ 000; scale bar: $50 \mathrm{~nm}$. m, mitochondrion; c, cytoplasm; r, cytoplasmic ribosomes; mn, nucleoid; om, outer mitochondrial membrane; im, inner mitochondrial membrane; *, some places where the nucleoid is connected to the surface of the crista by filaments. 
Four nucleoids in one mitochondrion section are shown in Fig. 7B. This micrograph documents how it can be difficult to recognize some nucleoids. Less intensive contrast might be the consequence of lower nucleoid condensation, which could be related to activity such as, for example, transcription (Shoubridge 2002).

The mitochondrial nucleoid shows a similar appearance to a perichromatin granule (Fig. $8 \mathrm{~A}$ ). The perichromatin granule is surrounded with a well-defined halo and can therefore be more easily found than most of the nucleoids. It can serve as a clue for learning about searching for nucleoids. Enlargement of the nucleoid and perichromatin granule shows that the fine structure of the filaments is different (Fig. 8B).

\section{Nucleoids in the mitochondria of MT1 human leukemia cells}

Two nucleoids located in the mitochondrion far from any membranes are shown in Fig. 9. The nucleoids in MT1 cells show a more granular and less condensed appearance than those in mouse leukemia cells or human Caco-2 cells.

Other electron-dense structures inside mitochondria that could be erroneously considered to be mitochondrial nucleoids

Some different electron-dense objects can be found inside mitochondria. These occur in normally growing cells at a much lower incidence than mitochondrial nucleoids occur. The detailed structure of such objects is not seen on the fluorescent screen of the electron microscope as clearly as on the photograph and, consequently, they could be erroneously considered to be nucleoids.

On occasion, convoluted membranes of cristae (probably lipid damages), resemble nucleoids as it can be seen in Fig. $10 \mathrm{~A}$. In the case of lower resolution use, these would not have been correctly identified. Unequivocal determination of these objects is allowed by setting the microscope for high resolution.

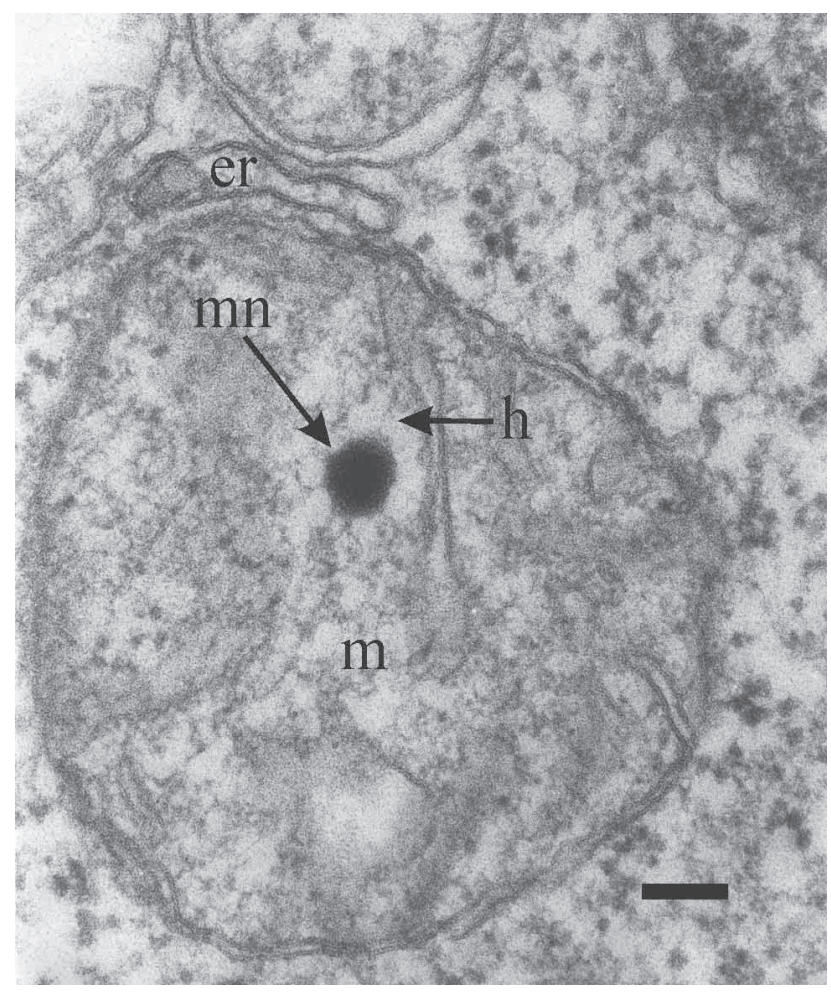

Figure 5. Section through the mitochondrion of a L1210 cell starving for serum showing condensed nucleoid and a halo around it. m, mitochondrion; $\mathrm{mn}$, strongly electron dense nucleoid surrounded with a halo (h); er, endoplasmic reticulum. Magnification: $\times 60000$; scale bar: $100 \mathrm{~nm}$.

Another example of the appearance of an electrondense formation is a section cut in parallel with a crista surface. Phospholipid heads bind contrasting agents and, if a section is just above the surface, that part appears electron-dense. Such sections usually occupy a larger diameter or surface than that of the nucleoid diameter. Usually, the section is not fully parallel with the crista surface and,

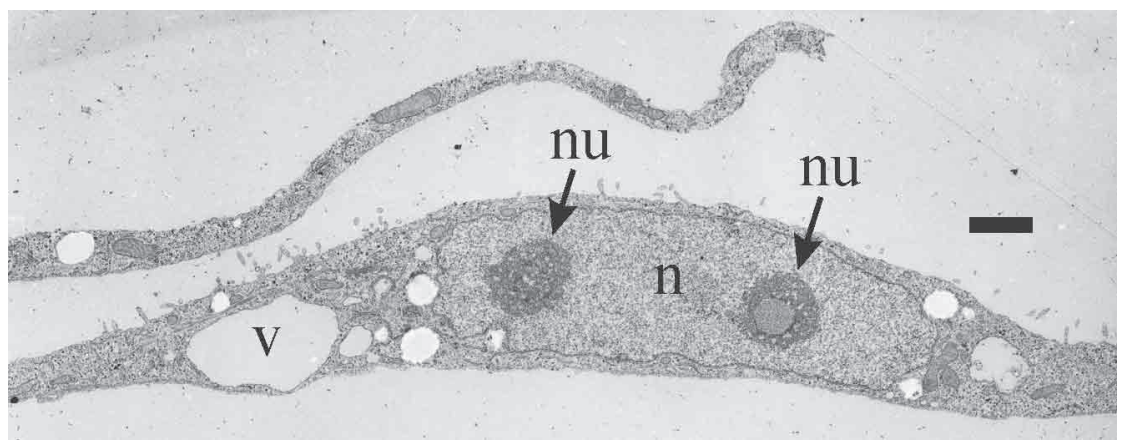

Figure 6. The view of human Caco-2 cell. Upper: section through periphery of flat cell; lower: section through the middle of the other cell. $\mathrm{n}$, nucleus; nu, nucleolus; $\mathrm{v}$, vacuole or vacuole-like cistern; respectively. Magnification: $\times 2$ 000: scale bar: $2 \mu \mathrm{m}$. 

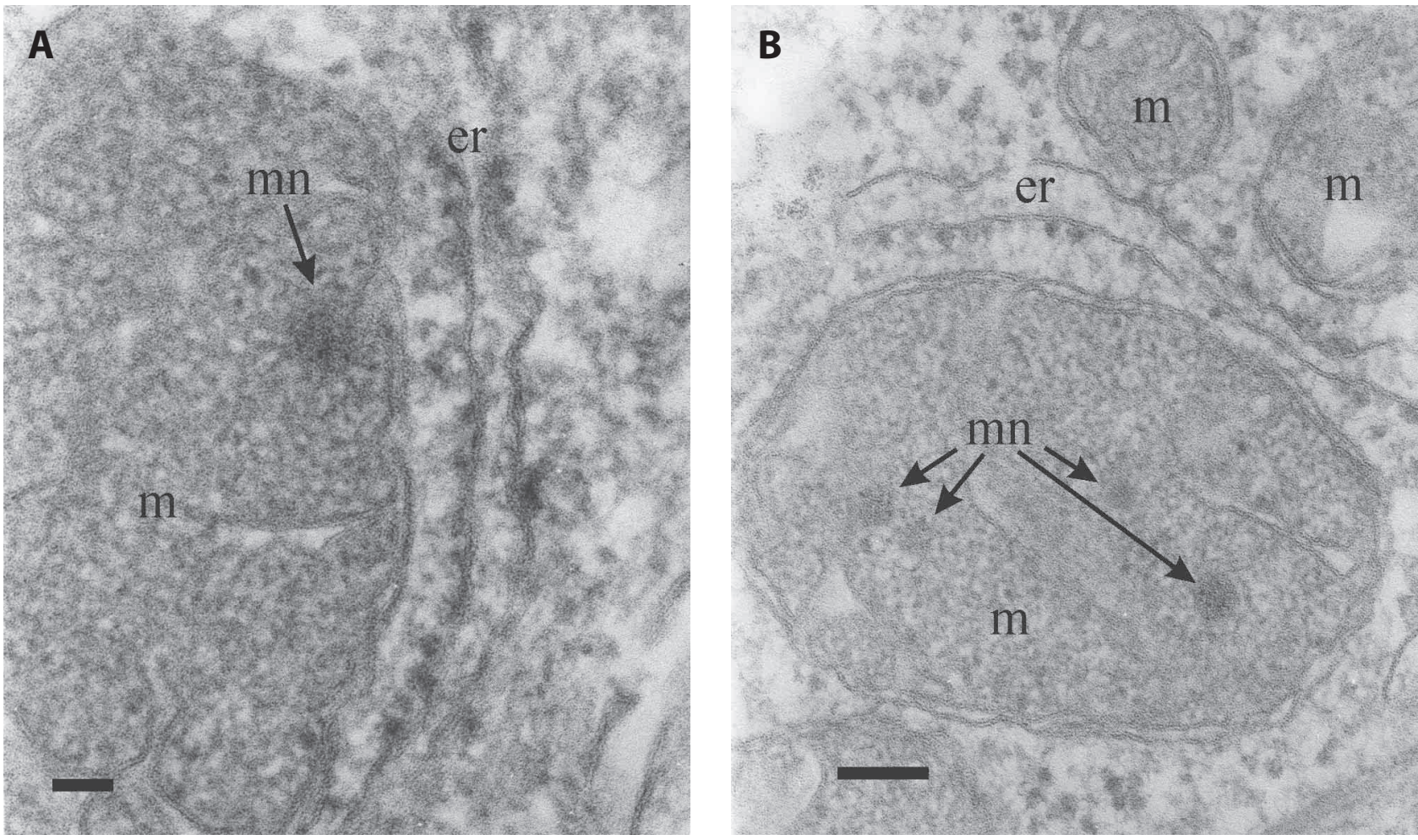

Figure 7. Observing of nucleoids in the Caco-2-cell mitochondria. A. Part of the cell mitochondrion showing nucleoid observed on much darker background than that in mouse cells. Nucleoid is anchored or attached to mitochondrial membranes. Rough endoplasmic reticulum envelopes the mitochondrion from the right side. Magnification: $\times 100$ 000; scale bar: $50 \mathrm{~nm}$. B. Caco-2 cell section showing several mitochondria and the endoplasmic reticulum. In mitochondrion are localized four barely distinguishable nucleoids. Magnification: $\times 75$ 000; scale bar: $100 \mathrm{~nm}$. m, mitochondrion; mn, nucleoids; er, endoplasmic reticulum.

consequently, some ribosomes "sitting" on the surface are also sectioned. Usually, these electron-dense regions are less electron-dense than nucleoids (Fig. 10B). The correct determination of these objects is again carried out by setting the microscope for high resolution, like in the case of convoluted membranes.

The observation of such objects in order to distinguish them is much more effective on a photograph than on the fluorescent screen.

We found, but rarely, very dense small "bullets" inside the mitochondria. Such mitochondria had a light matrix and, within a given cell, more mitochondria showed signs of degenerative processes. The small bullets are probably mitochondrial nucleoids in a certain stage of degeneration (Fig. 10C).

\section{Searching for nucleoid division}

Electron microscopically-documented division of a rod shaped large nucleoid of Physarum has been published (Kuroiwa et al. 2006). The nucleoid's fission is character- ized by changing shape to a dumbbell-shape; this also represents a stage of dividing mitochondria because these processes run at the same time. The nucleoids split into two daughter nucleoids together with the splitting of the mitochondrion.

We have searched for dumbbell or elongated shapes of nucleoids but we have not seen any sign of nucleoid division in spite of observing several hundreds of mouse nucleoids and tens of nucleoids in two human cell lines. The impossibility of finding a different nucleoid shape than approximately spherical argues that the division of mammalian nucleoids is realized in a different way than that of Physarum. On the other hand, we have occasionally observed two or more nucleoids close together that could be the result of mitochondrial DNA (mtDNA) replication. Sometimes one of the two close nucleoids is smaller, so it could represent the daughter one, just finishing the stage of replication (Fig. 3). Also, on confocal images with labeled DNA, the observer can see nucleoids of different intensity and size close together, where smaller ones could represent the daughter ones. 


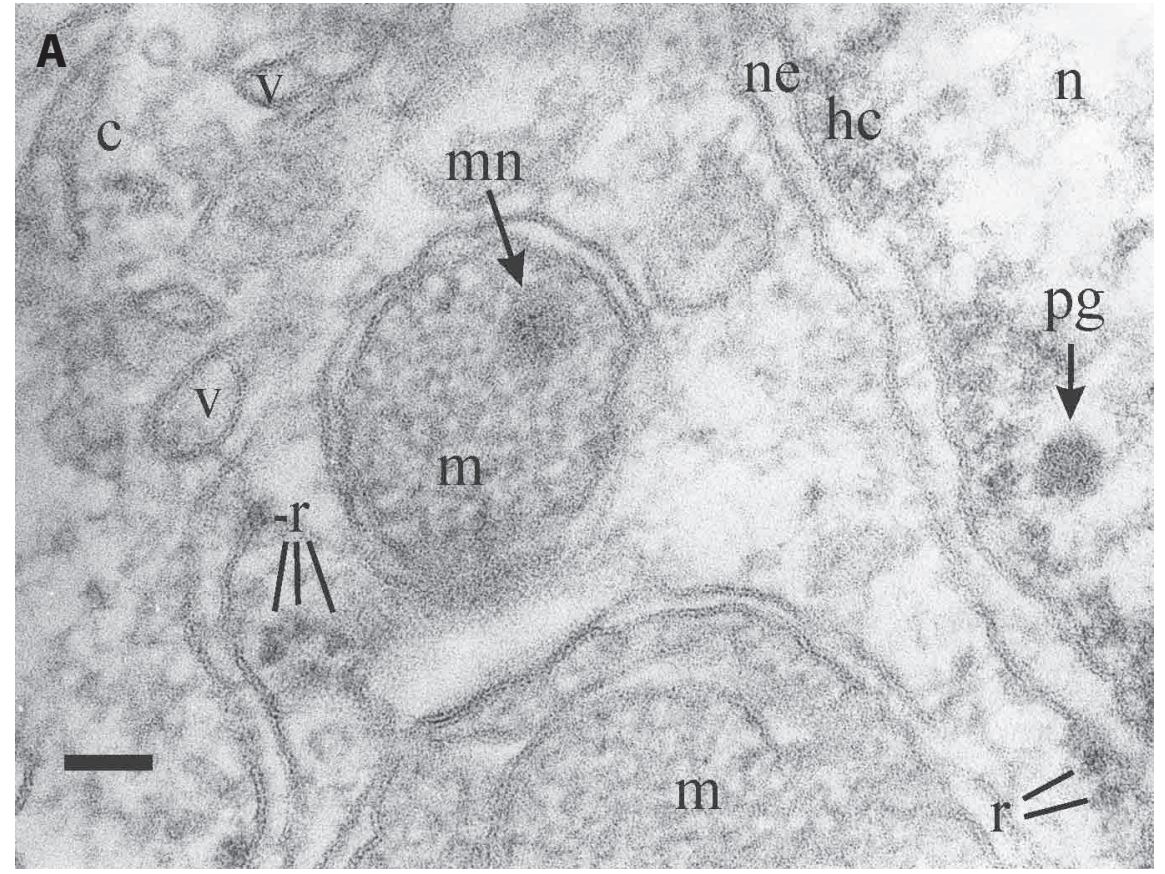

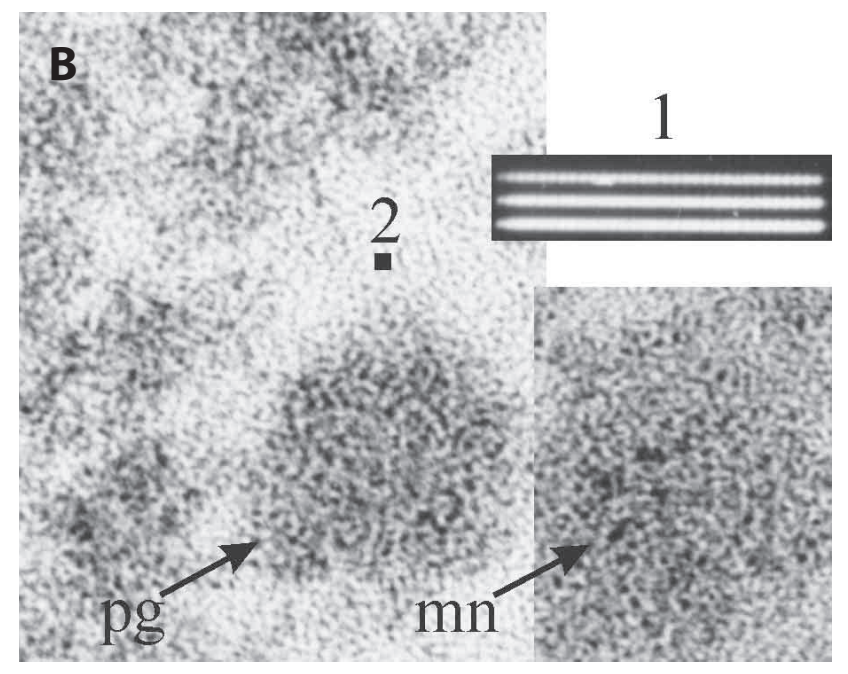

\section{Discussion}

Morphology and observability of nucleoids by transmission electron microscopy

In early electron microscopic studies, the DNA of mitochondria from many cell types were visualized by electron microscopy as fibrils of $2 \mathrm{~nm}$ diameter in electron transparent areas (Nass and Nass 1963). Subsequently, the same authors published work suggesting that these results could be influenced by the method used and they presented micrographs
Figure 8. Comparison of mitochondrial nucleoid and perichromatin granule morphology. A. Caco-2 cell section. Magnification: $\times 100000$; scale bar: $50 \mathrm{~nm}$. B. Enlargement of a perichromatin granule (left side) in comparison to the same degree enlargement of a mitochondrial nucleoid (right side). The insets are in the opposite order than the objects in Fig. 8A to better enable comparison of the fine structure. The strands in the perichromatin granule seem to be thinner than $2 \mathrm{~nm}$, but the space occupied by contrasting agents must be taken account. The strands in the mitochondrial nucleoid are thinner than those in the perichromatin granule. Magnification: $\times 100$ 000; scale bar 1: $50 \mathrm{~nm}$, bar 2: $2 \mathrm{~nm}$. c, cytoplasm; n, nucleus; v, various cytoplasmic vesicles; ne, nuclear envelope; hc, heterochromatin; $\mathrm{m}$, mitochondria; $\mathrm{mn}$, nucleoid; pg, perichromatin granule; $r$, cytoplasmic ribosomes.

of electron-dense nucleoids with a light halo in the matrix using modified method (Nass 1969).

As early as in 1969, nucleoids were identified as electrondense bodies. However, except these findings from 1969 and the well-characterized large nucleoid of Physarum (Suzuki et al. 1982; Kuroiwa et al. 2006), there were no published electron micrographs of mitochondrial nucleoids until now.

This situation gives rise to uncertainty about the behavior of mitochondrial nucleoids in transmission electron microscopy (for discussion of this theme, see the follow- 


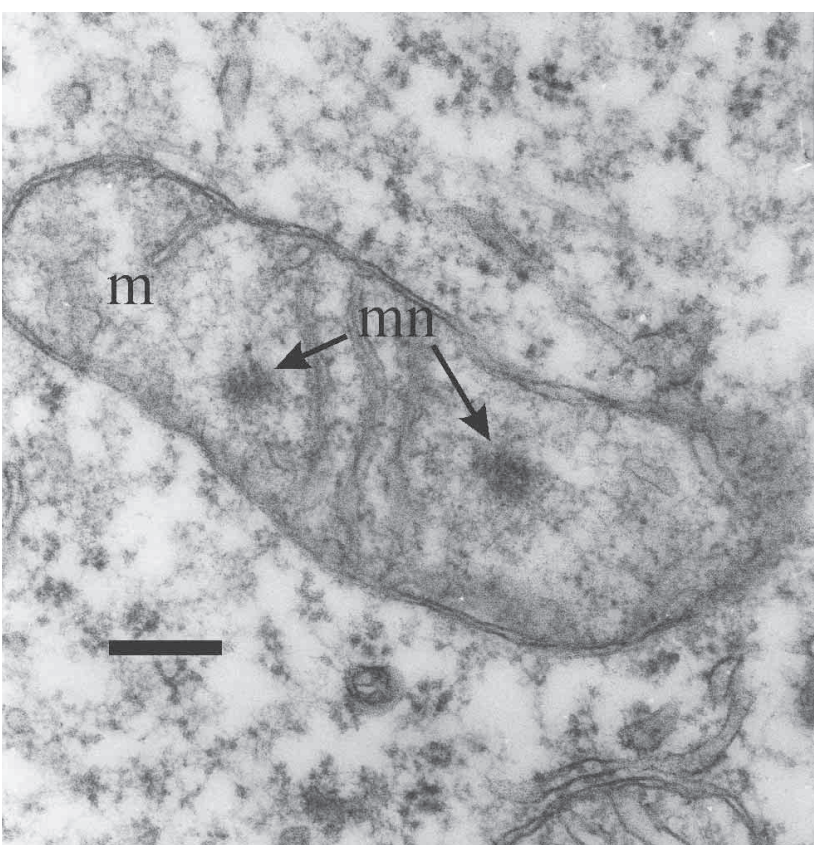

Figure 9. MT1 cell mitochondrion section containing two wellrecognizable nucleoids. They show a more granular and less condensed detailed structure than those in mouse leukemia cells or human Caco-2 cells but the general view is very similar. m, mitochondrion; mn, nucleoids. Magnification: $\times 40$ 000; scale bar: $200 \mathrm{~nm}$.

ing reviews and references therein: Kuroiwa et al. 2006; Malka et al. 2006). From many data obtained by confocal microscopy and molecular biology approaches, it was unequivocally determined that nucleoids are complexes of condensed DNA and proteins which must appear as electron-dense objects under the transmission electron microscope. From data published earlier (Nass 1969; Suzuki et al. 1982), data published recently (Kuroiwa et al. 2006) and our presented data, it is clear that appearance of nucleoids is not any exception to the rule that condensed DNA-protein complexes appear as electron-dense objects, similar to heterochromatin, if there is an appropriate contrasting method used. The same rule was documented in Physarum. Under the satisfactory contrasting, the only problem is in finding of small objects of similar electron density like a density of surroundings. We usually used the magnification setting of $12000 \times$ plus zoom $10 \times$ (searching on a small screen only). Searching for nucleoids is very laborious and requires patience and training; in addition, it is beneficial to have experience with searching for other small objects like small viruses.

In summary, mammalian nucleoids are small objects (easily escape notice of the observer) with diameters usually ranging from $60 \mathrm{~nm}$ to $90 \mathrm{~nm}$, i.e. only three or four times larger than the diameter of the cytoplasmic ribosome. A nucleoid is smaller than a retrovirus. On the other hand, in some cells, it is less difficult to find nucleoids (in our search, there were the L1210 mouse cells presented in this paper, and mouse L cells were shown by Nass 1969). It is very probable that more observers noticed these small objects in the mitochondria. However, without adequate sample preparation, a microscope setting for high resolution and identification of the fibrillar structure, the small dots could be considered to be matrix granules or some other small dense particles (Barnard 1981). Comparison of the fine fibrils in the objects observed in our laboratory with the published ones of Physarum nucleoids assured us that the objects are of the same nature.

Surprisingly, nucleoids in different cell types do not demonstrate the same absolute appearance. They are generally electron-dense, but if the mitochondrial matrix is also relatively dense, like in case of the human Caco- 2 cell line, these objects can easily evade observation. In the case of the human lymphoblastoid cell line MT1, nucleoids are not as dense as in L1210 cells and Caco- 2 cells. The MT1 cell line is slowly growing and mismatch repair deficient and it could have even more defects in the genome. However, these features need not to be in any connection to slightly different morphology of mitochondrial nucleoid. We only notify that observer can meet some small variation in morphology of nucleoids in different cells.

In L1210 cells starving for serum, the matrix is lighter and, consequently, the nucleoids are more easily found. Some of the nucleoids are also more packed than in the cells cultured in the presence of serum.

We recommend starting to learn to find nucleoids with the L1210 cell line or L mouse cells. The L1210 mouse cell line would be also the best model for studying nucleoid replication and division.

\section{Why has electron microscopy documentation of the} mitochondrial nucleoids of higher eukaryotes not been presented for tens of years, and why have electron micrographs of human mitochondrial nucleoids not been published at all so far?

The first reason could be inadequate sample preparation. From a methodological point of view, we consider the most important step to be the contrasting of the sample - the contrasting of the cells en bloc overnight in a uranyl acetate solution before embedding. This step considerably increases the resulting contrast of many of the structures in the section. Strongly contrasted thin sections help to distinguish nucleoids from the matrix.

Second, it was much more difficult to find nucleoids in the two human cell lines that we have used than in the mouse cell line. The situation could be similar in some other human 

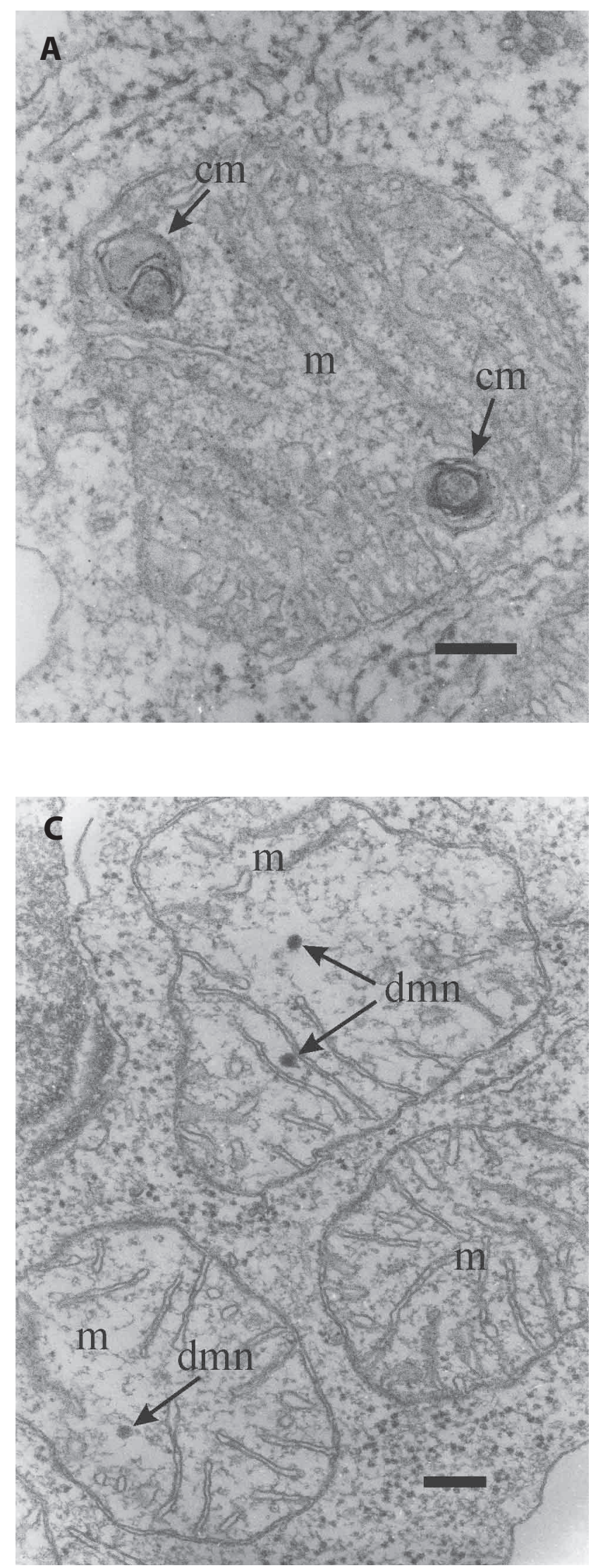

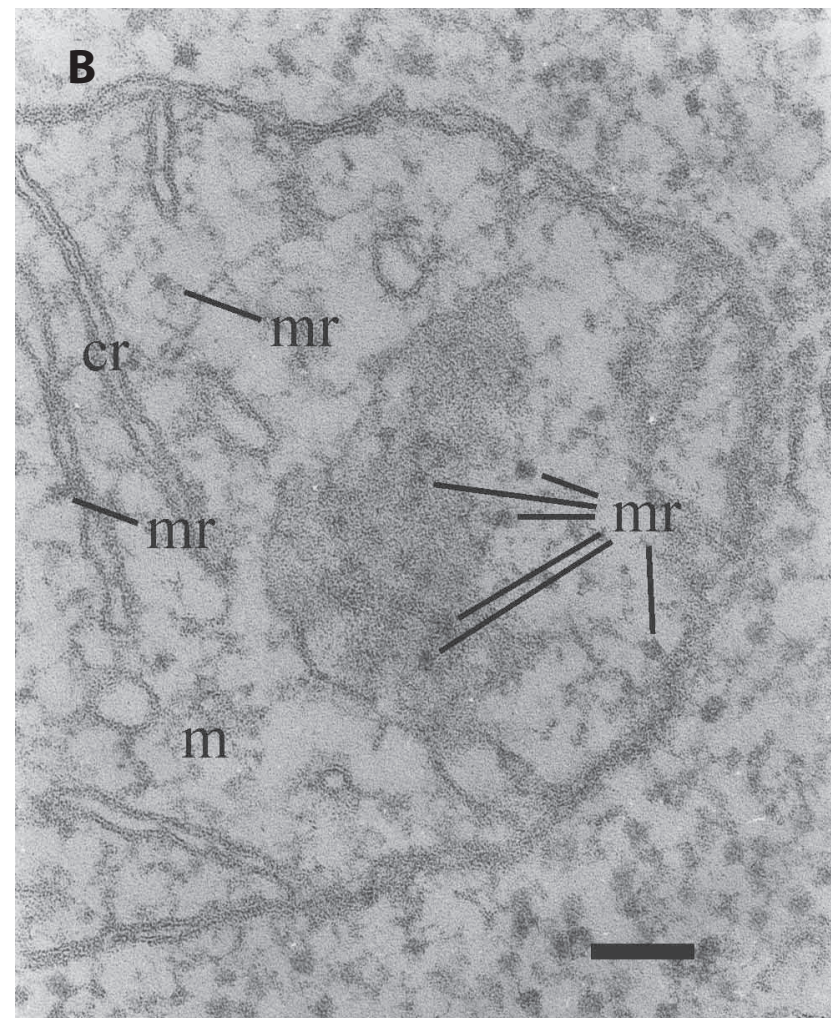

cells; human cells are practically the sole model of higher eukaryotes used at present.

Third, the researcher must be prepared to search for very small objects that are located in relatively electrondense surroundings. Therefore, to obtain a light mitochondrial matrix and subsequent better visualization of nucleoids, preparation of thin sections is needed. If the section is thicker, the matrix acquires a very strong electron density, even when only one side of the section is contrasted.

Figure 10. Various electron-dense structures inside mitochondria that could be erroneously considered to be nucleoids. A. MT1 cell mitochondrion section containing convoluted membranes that remind nucleoids. Magnification: $\times 40$ 000; scale bar: $200 \mathrm{~nm}$ B. Section of the L1210 cell, parallel with the surface of one crista, containing the electron-dense area which can mimic a nucleoid (but in this case it represents much larger area than nucleoids do). Mitochondrial ribosomes ( $\mathrm{mr}$ ) are positioned mainly on the crista surface. Magnification: $\times 75$ 000; scale bar: $100 \mathrm{~nm}$ C. Part of the L1210 cell showing three mitochondria. In two of them very rare findings of small dark dots are observed representing probably degenerated mitochondrial nucleoids. Magnification: $\times 30$ 000; scale bar: $200 \mathrm{~nm}$. m, mitochondrion; $\mathrm{cm}$, convoluted membranes; $\mathrm{cr}$, one of the mitochondrial cristae; $\mathrm{dmn}$, degenerated nucleoids. 
Fourth, a proper strategy for setting an electron microscope to obtain maximal resolving power is a prerequisite for success in searching for nucleoids ${ }^{1}$.

It is interesting to compare searches for nucleoids by means of electron and fluorescent microscopy. Fluorescent microscopy has a much lower resolution but, in one cell, hundreds of small spots of nucleoids shine on a very dark surrounding; therefore, it is very easy to recognize them. On the other hand, with the electron microscope, we have to suppose that we search for only one or two small objects in one "large" section among many other dense objects and in relatively dense surroundings (in many cases there is no nucleoid in a given section).

\section{Proportion of nucleoids bound to the inner mitochondrial surface}

About $80 \%$ of the nucleoids in the mitochondria in our preparations appear far from the inner surface (see also Nass 1969), but this number is overestimated for technical reasons. In some cases, the nucleoid can be very close to the surface but the section is cut in such a direction that the nucleoid appears to be very far from the membranes. Even if the nucleoid is far from the surface, we can frequently trace some filaments directed towards the surface membranes. The question arises if all nucleoids are not anchored to the mitochondrial surface at a distance. On the other hand, it is not possible to find any connection to the surface of some nucleoids (e.g. Figs. 2A,B, 3A, 9). This situation could correspond to the presence of two subsets of nucleoids with different sedimentation velocities (Wang and Bogenhagen 2006). We have found more nucleoids bound to the surface in cells that enlarge their volumes, i.e. cells in mitosis or preparing for mitosis. This could correspond to some activity of the nucleoid; for example, it could be related to replication.

\section{Presence of a halo surrounding nucleoids}

Some nucleoids are surrounded with a light halo but some are not, even in the same section of mitochondria. This

\footnotetext{
${ }^{1}$ Nucleoids in the human cells documented in the micrographs in this work seem to be very well distinguished from the surroundings, but the reason for this is also in the manipulation between the microscope and the end micrograph. The film has much higher contrast than that which the observer can see on the luminescent screen. The developer intensifies the contrast of the film similarly as the developer on the photographic paper. Even photographic papers used in this work were contrast papers. Therefore, there were several steps that increased the contrast of the already photographed objects. In brief, it is very easy to find a nucleoid on the photograph, but much more difficult to find it on the fluorescent screen.
}

feature can represent certain stages of nucleoid activity. Electron-dense regions in the nucleus represent heterochromatin and light regions represent euchromatin. Light regions in this case represents unpacked DNA - the subject of transcription. We have noticed that the presence of the halo surrounding nucleoids is more often found in cells starving for serum and that nucleoids are highly packed. Therefore, we suppose that the presence of the halo represents a non-active stage of the nucleoid. However, we have still found occasions of mitoses in cells starving for serum for $24 \mathrm{~h}$ (not shown); thus, longer starvation (e.g. $48 \mathrm{~h}$ ) would give more valuable results.

\section{Collaboration of cytoplasmic and mitochondrial ribosomes}

It has been suggested that 1) the mitochondrial nucleoid is an organizing center for the transcription and translation of mitochondrial proteins and the transcription of mitochondrial RNAs; 2) mitochondrial ribosomes are localized close to nucleoids on the inner membrane of the mitochondrion and that this is where they perform translation; 3 ) the cytoplasmic ribosomes would be localized on the opposite side of the mitochondrial surface, on the outer mitochondrial membrane. From those presumptions, it has also been suggested that cytoplasmic and mitochondrial ribosomes collaborate spatially.

For that reason, we concentrated on the search for situations where cytoplasmic and mitochondrial ribosomes are close together on either side of the mitochondrial membrane. We have found that mitochondrial ribosomes are generally quite evenly distributed on the surfaces of the cristae (Fig. 10B and 11). Some mitochondrial ribosomes are also present on the inner membrane (not on the cristae), but can only be found on rare occasions in close proximity to cytoplasmic ribosomes. Conversely, the cytoplasmic ribosomes that are sitting on the outer mitochondrial membrane generally do not have a counterpart from inside the mitochondrion. Hence, if a spatial collaboration between mitochondrial ribosomes and cytoplasmic ribosomes exists, it would be a very rare event because most of the mitochondrial ribosomes are positioned on the cristae.

Mitochondrial ribosomes do not show any concentration gradient at a short distance from the nucleoid in the electron micrographs. Hence, translation on mitochondrial ribosomes is probably performed close to the required location, to which cytoplasmic proteins are transported.

\section{Association of nucleoids with the cytoskeleton}

Models of a molecular system enabling interactions across both mitochondrial membranes have been proposed (Chen and Butow 2005). Biochemical studies of mtDNA nucleoids in higher eukaryotes have reported their association with the inner membrane (Garrido et al. 2003) and with cytoskeletal structures (Wang and Bogenhagen 2006). Therefore, we also 
searched for the contact of the cytoskeleton with those nucleoids having contact with the surface of the mitochondrion.

The term cytoskeleton has a very wide definition and covers many different fibrils and microtubules. All "free" cytoplasmic ribosomes are bound together through a network of fine fibrils - the cytoskeleton (Zambetti et al. 1990). These proteinous fibrils appear faintly or as shadows in the electron microscope. These fibrils not only connect ribosomes together, but they also connect ribosomes in the vicinity of the mitochondrion to the outer mitochondrial membrane. The same fibrils (or fibrils of the same appearance) can be observed on the outer surface of the mitochondrion at the place where the nucleoid is bound to the inner surface of the mitochondrion (see also Iborra et al. 2004). These fibrils are also directed to the nearest cytoplasmic ribosomes. With regard to this latter phenomenon, one can speculate that such a cytoskeleton can serve for protein transport to the place of need. In the case of mediating contact of the ribosomes with the outer mitochondrial membrane, it might also realize the flow of energy from the mitochondrion, or it might represent both functions at once.

Is the mouse cell line L1210 a suitable model for the observation of nucleoids in various conditions or under various influences?

We have searched for mitochondrial nucleoids in three cell lines. We have most easily found nucleoids in the mouse leukemia cell line L1210 starving for serum. The condensed nucleoids were most distinguishable against the light background of the mitochondrial matrix in these cells. High packaging of the nucleoids in cells starving for serum is probably the effect of remodeling in response to metabolic conditions (Kucej et al. 2008). In L1210 cells cultured in the presence of serum, the mitochondrial matrix is more electron-dense as a result of mitochondrial activity, but nucleoids are still very well discernable. This cell line is easily cultured, has a short doubling time and we consider it an ideal model for these purposes.

\section{Suitability of the human cell lines used as model systems}

For the two human cell lines used, it was difficult to find nucleoids, and this identification process therefore required longer training. It is even probable that some nucleoids evaded the attention of the observer, regardless of effort. Therefore, if these cell lines are used, we recommend not using them exclusively, but in comparison with mouse cell lines. Both human cell lines used are slow growing. It would be beneficial to test some other human leukemia cell lines with short doubling times and in the early exponential phase of growth. Caco- 2 cells are strongly proteosynthetically active. Such cells utilize substantial energy for proteosynthesis and, as a consequence, the mitochondrial matrix is electron-dense. Therefore, the better model would be

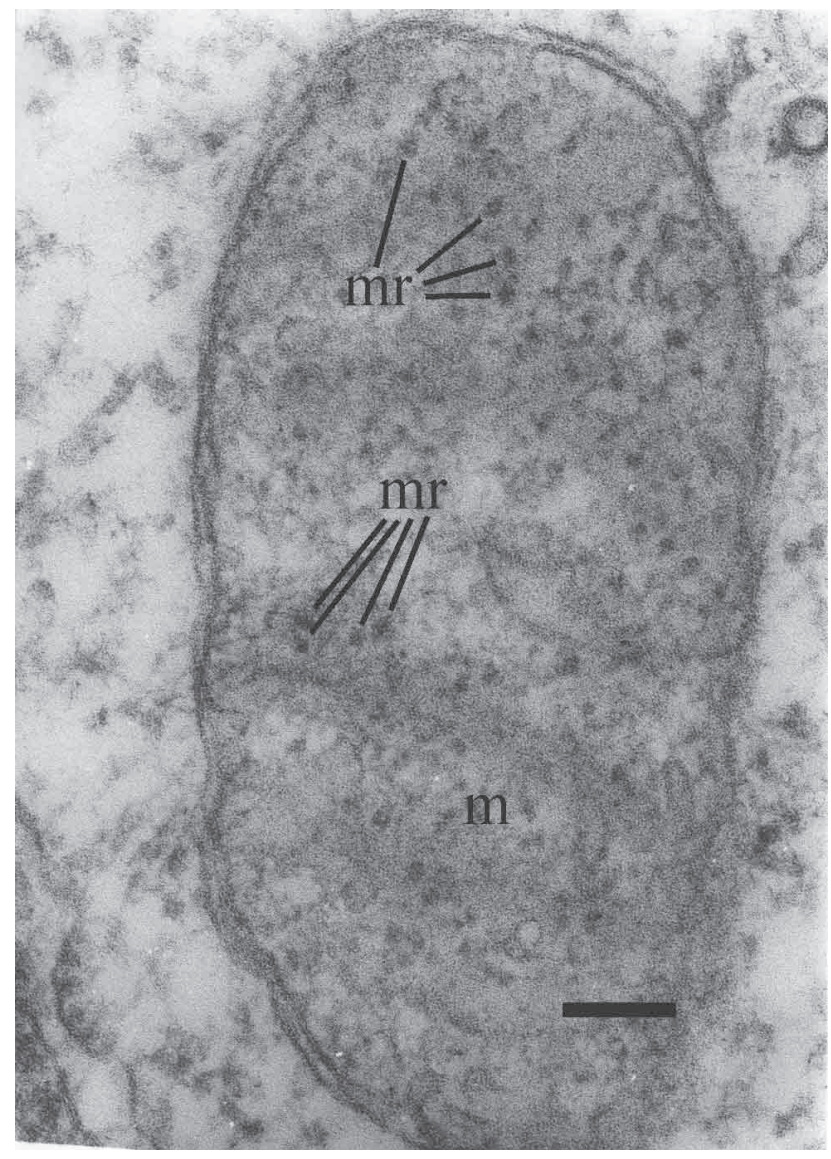

Figure 11. Demonstration of random distribution of mitochondrial ribosomes in MT1 cell mitochondrion section that is parallel to the surfaces of mitochondrial cristae. Some mitochondrial ribosomes are marked. $\mathrm{m}$, mitochondrion; $\mathrm{mr}$, mitochondrial ribosomes. Magnification: $\times 75$ 000; scale bar: $100 \mathrm{~nm}$.

a cell line with minimal external protein production, similar to the mouse leukemia cell line.

It would be very useful starting searching with perichromatin granules for learning how to find nucleoids in human cells. Perichromatin granules show a very similar appearance to nucleoids, with only the fine structure being different (Fig. 8).

We must always consider that mitochondrial nucleoids are very small objects. To emphasize how small the nucleoid is, we also present a micrograph where two centrioles are close to a mitochondrion with a nucleoid (Fig. 12).

In summary, in comparison to the mouse leukemia cell line L1210, the two human cell lines used in this work are not an ideal model for monitoring nucleoid behavior after some specific treatment, and we recommend the search for a more suitable human cell line. Nevertheless, the positive result is that the different positions and appearances of 


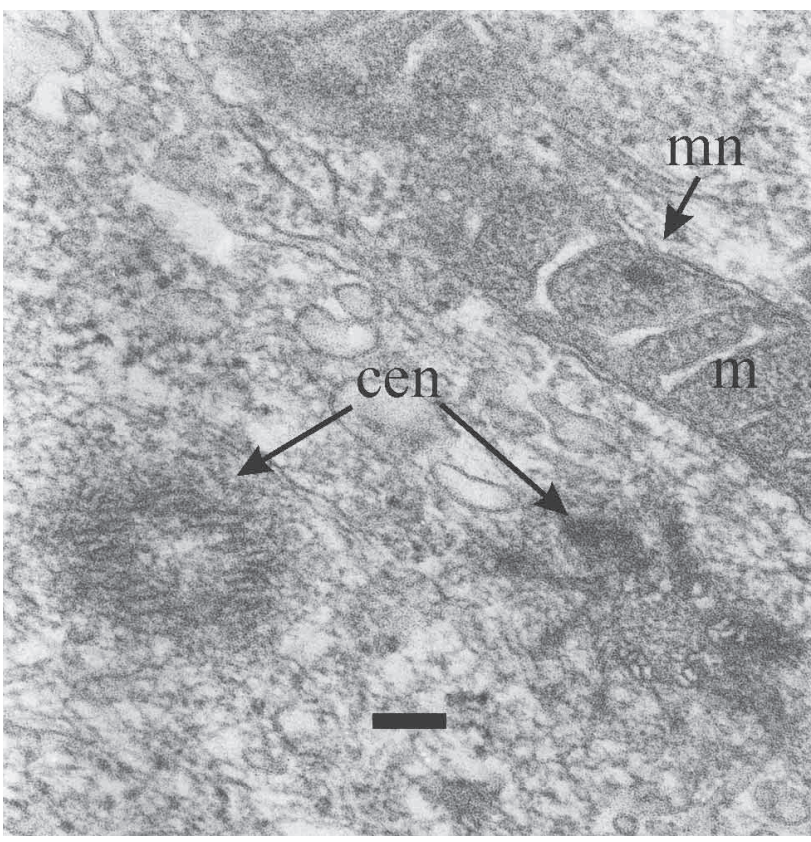

Figure 12. Size comparison of centrioles and nucleoid. Part of the Caco- 2 cell mitochondrion in close proximity to centrioles showing a nucleoid anchored to the surface mitochondrial membranes. $\mathrm{m}$, mitochondrion; mn, nucleoid; cen, centrioles.

Magnification: $\times 50000$; scale bar: $100 \mathrm{~nm}$.

the nucleoids presented in this paper are in full agreement with the idea that nucleoids exhibit very dynamic behavior evidently dependent on their various functional stages.

The relationship between the functional stage and the position of nucleoids inside mitochondria

We have observed that about $80 \%$ of nucleoids in mouse leukemia cells were located inside mitochondria, far from the inner mitochondrial surface. This leukemia cell line represents rapidly proliferating cells, but it was taken for observation after the exponential growth phase when the increase of cellular mass is much lower than in the early exponential growth. Despite this, we have noticed that the cells that show and increasing of cellular mass - cells preparing for mitosis or cells in mitosis - very often exhibit nucleoids close to or bound to the surface membranes.

Based on these observations, we can speculate the position of the nucleoid being in contact with the mitochondrial surface occurs in direct relation to the growth of the cell and, therefore, can represent the replication stage of the nucleoid.

In conclusion, the position and the nucleoid appearance can be the result of highly dynamic behavior and can correspond to different stages for the nucleoid, such as replica- tion, transcription and the "sleeping" state (see for example Bogenhagen et al. 2008).

Collaboration of molecular biology and electron microscopy in the investigation of mitochondrial nucleoids

How electron microscopy can contribute to the molecular biology research. The main advantage of electron microscopy is the ability to establish location of different objects in conditions of high resolution. Previously some authors observed mitochondrial nucleoids being linked to the proteinaceous structure spanning both the outer and inner mitochondrial membranes (Kondo-Okamoto et al. 2003; Meeusen and Nunnari 2003). More recently, it has been reported that the nucleoids are linked to protein folding machinery and metabolic enzymes at the mitochondrial inner membrane (Wang and Bogenhagen 2006). Our micrographs confirm both possibilities which exist in the mitochondrion at the same time. One part of nucleoids is linked to inner membranes of cristae, the other part is attached to the complexes spanning the outer and inner mitochondrial membranes. It would not be easy to show these both kinds of attachment at the same time by the molecular biology techniques. We have also shown that the structure which spans both mitochondrial membranes does not only penetrate them, but in most cases substitutes membranes in relatively large area. This structure is complex and the absence of phospholipids may facilitate very important functions, for example effective transport.

Different condensation of nucleoids clearly corresponds to their activity. The nucleoids in the cells starving for serum are less active or inactive and more condensed. Poorly contrasted nucleoids in the active cells most probably reflect transcription. Such information could be obtained by molecular biology techniques; direct observation is valuable, however, the combination of both approaches would be unambiguous.

It can be easily shown by electron microscopy where the replication and transcription of the nucleoid take place by the appropriate adjusting of growth conditions of cell cultures. It would be difficult to get such information by molecular biology techniques.

These examples show that transmission electron microscopy can offer very important and often irretrievable information for simplifying and directing molecular biology approaches. The collaboration of both techniques can substantially help to our understanding of mitochondrial genome functioning.

\section{Conclusion and future directions}

This work was especially dedicated to the search for nucleoids and the basic characterization of them and it is mostly phenomenological in nature. The results are based mostly 
on observations, and most of the conclusions are based on the comparison of micrographs with other authors' results obtained by different, especially molecular biology methods. We would like to express a suggestion that the comparison of rapidly growing cells in the early exponential phase in the presence of $10 \%$ fetal bovine serum with those cultured in the presence of $1 \%$ fetal serum and cells starved for serum for $48 \mathrm{~h}$ might provide even more valuable results. The work provides information on how to search for nucleoids and suggests how to avoid certain obstacles while searching. Mitochondrial nucleoids are described morphologically in detail in this work, and, at this stage, this approach can be combined with many different experimental approaches.

Present work can be the start of the characterization of the mitochondrial nucleoid and its functions at a considerably higher resolution than that enabled by the methods of confocal microscopy.

Acknowledgments. This investigation has been supported by the Slovak Cancer Research Foundation. We are grateful to Mrs. Margita Šulíková and Miss Gabriela Gašajová for providing cell cultures.

\section{References}

Barnard T. (1981): Mitochondrial matrix granules, dense particles and the sequestration of calcium by mitochondria. Scan. Electron. Microsc. 419-433

Bogenhagen D. F., Rousseau D., Burke S. (2008): The layered structure of human mitochondrial DNA nucleoids. J. Biol. Chem. 283, 3665-3675; doi:10.1074/jbc.M708444200

Chen X. J., Butow R. A. (2005): The organization and inheritance of the mitochondrial genome. Nat. Rev. Genet. 6, 815-825; doi:10.1038/nrg1708

Chen X. J., Wang X., Butow R. A. (2007): Yeast aconitase binds and provides metabolically coupled protection to $\mathrm{mi}-$ tochondrial DNA. Proc. Natl. Acad. Sci. U.S.A. 104, 13738-13743; doi:10.1073/pnas.0703078104

Garrido N., Griparic L., Jokitalo E., Wartiovaara J., van der Bliek A. M., Spelbrink J. N. (2003): Composition and dynamics of human mitochondrial nucleoids. Mol. Biol. Cell 14, 1583-1596; doi:10.1091/mbc.E02-07-0399

He J., Mao C. C., Reyes A., Sembongi H., Di Re M., Granycome C., Clippingdale A. B., Fearnley I. M., Harbour M., Robinson A. J., Reichelt S., Spelbrink J. N., Walker J. E., Holt I. J. (2007): The AAA+ protein ATAD3 has displacement loop binding properties and is involved in mitochondrial nucleoid organization. J. Cell. Biol. 176, 141-146; doi:10.1083/jcb.200609158

Holt J., He J., Mao C. C., Boyd-Kirkup J. D., Martinsson P., Sembongi H., Reyes A., Spelbrink J. N. (2007): Mammalian mitochondrial nucleoids: Organizing an independently minded genome. Mitochondrion 7, 311-321; doi:10.1016/ j.mito.2007.06.004
Iborra F. J., Kimura H., Cook P. R. (2004): The functional organization of mitochondrial genomes in human cells. BMC Biol. 2, 9; doi:10.1186/1741-7007-2-9

Kaufman B. A., Durisic N., Mativetsky J. M., Costantino S., Hancock M. A., Grutter P., Shoubridge E. A. (2007): The mitochondrial transcription factor TFAM coordinates the assembly of multiple DNA molecules into nucleoid-like structures. Mol. Biol. Cell 18, 3225-3236; doi:10.1091/ mbc.E07-05-0404

Kondo-Okamoto N., Shaw J. M., Okamoto K. (2003): Mmm1p spans both the outer and inner mitochondrial membranes and contains distinct domains for targeting and foci formation. J. Biol. Chem. 278, 48997-49005; doi:10.1074/jbc. M308436200

Kucej M., Kucejova B., Subramanian R., Chen X. J., Butow R. A. (2008): Mitochondrial nucleoids undergo remodeling in response to metabolic cues. J. Cell. Sci. 121, 1861-1868; doi: $10.1242 /$ jcs. 028605

Kuroiwa T. (1973): Studies on mitochondrial structure and function in Physarium polycephalum. I. Fine structure, cytochemistry, and $3 \mathrm{H}$-uridine autoradiography of a central body in mitochondria. Exp. Cell Res. 78, 351-359; doi:10.1016/0014-4827(73)90079-7

Kuroiwa T., Nishida K., Yoshida Y., Fujiwara T., Mori T., Kuroiwa H., Misumi O. (2006): Structure, function and evolution of the mitochondrial division apparatus. Biochim. Biophys. Acta 1763, 510-521; doi:10.1016/j.bbamcr.2006.03.007

Legros F., Malka F., Frachon P., Lombès A., Rojo M. (2004): Organization and dynamics of human mitochondrial DNA. J. Cell. Sci. 117, 2653-2662; doi:10.1242/jcs.01134

Macierzanka M., Plotka M., Pryputniewicz-Drobinska D., Lewandowska A., Lightowlers R., Marszalek J. (2008): Maintenance and stabilization of mtDNA can be facilitated by the DNA-binding activity of Ilv5p. Biochim. Biophys. Acta 1783, 107-117; doi:10.1016/j.bbamcr.2007.09.009

Malka F., Lombès A., Rojo M. (2006): Organization, dynamics and transmission of mitochondrial DNA: focus on vertebrate nucleoids. Biochim. Biophys. Acta 1763, 463-472; doi:10.1016/j.bbamcr.2006.04.001

Meeusen S., Nunnari J. (2003): Evidence for a two membrane-spanning autonomous mitochondrial DNA replisome. J. Cell Biol. 163, 503-510; doi:10.1083/jcb.200304040

Nass M. M. (1969): Mitochondrial DNA. I. Intramitochondrial distribution and structural relations of single- and double-length circular DNA. J. Mol. Biol. 42, 521-528; doi:10.1016/0022-2836(69)90240-X

Nass M. M., Nass S. (1963): Intramitochondrial fibers with DNA characteristics. I. Fixation and electron staining reactions. J. Cell Biol. 19, 593-611; doi:10.1083/jcb.19.3.593

Nosek J., Tomaska L., Bolotin-Fukuhara M., Miyakawa I. (2006): Mitochondrial chromosome structure: an insight from analysis of complete yeast genomes. FEMS Yeast Res. 6, 356-670; doi:10.1111/j.1567-1364.2005.00016.x

Prachar J. (2003): Intimate contacts of mitochondria with nuclear envelope as a potential energy gateway for nucleo-cytoplasmic mRNA transport. Gen. Physiol. Biophys. 22, 525-534

Sasaki N., Kuroiwa H., Nishitani C., Takano H., Higashiyama T., Kobayashi T., Shirai Y., Sakai A., Kawano S., Murakami- 
Murofushi K., Kuriowa T. (2003): Glom is a novel mitochondrial DNA packaging protein in Physarum polycephalum and causes intense chromatin condensation without suppressing DNA functions. Mol. Biol. Cell 14, 4758-4769; doi:10.1091/mbc.E03-02-0099

Shadel G. S., Clayton D.A. (1997): Mitochondrial DNA maintenance in vertebrates. Annu. Rev. Biochem. 66, 409-435; doi:10.1146/annurev.biochem.66.1.409

Shoubridge E. A. (2002): The ABCs of mitochondrial transcription. Nat. Genet. 31, 227-228; doi:10.1038/ng0702-227

Sumitani M., Kasashima K., Ohta E., Kang D., Endo H. (2009): Association of a novel mitochondrial protein M19 with mitochondrial nucleoids. J. Biochem. 146, 725-732; doi:10.1093/jb/mvp 118

Suzuki T., Kawano S., Kuroiwa T. (1982): Structure of three-dimensionally rod-shaped mitochondrial nucleoids isolated from the slime mould Physarum polycephalum. J. Cell. Sci. 58, 241-261

Wang Y., Bogenhagen D. F. (2006): Human mitochondrial DNA nucleoids are linked to protein folding machinery and metabolic enzymes at the mitochondrial inner membrane. J. Biol. Chem. 281, 25791-25802; doi:10.1074/jbc. M604501200

Zambetti G., Wilming L., Fey E. G., Penman S., Stein J., Stein G. (1990): Differential association of membrane-bound and non-membrane-bound polysomes with the cytoskeleton. Exp. Cell Res. 191, 246-255; doi:10.1016/00144827(90)90011-X

Received: July 9, 2009

Final version accepted: January 11, 2010 